\title{
SECOND-ORDER ESTIMATES FOR BOUNDARY BLOWUP SOLUTIONS OF SPECIAL ELLIPTIC EQUATIONS
}

\author{
CLAUDIA ANEDDA, ANNA BUTTU, AND GIOVANNI PORRU
}

Received 20 October 2005; Accepted 7 November 2005

We find a second-order approximation of the boundary blowup solution of the equation $\Delta u=e^{\left.u|u|\right|^{\beta-1}}$, with $\beta>0$, in a bounded smooth domain $\Omega \subset R^{N}$. Furthermore, we consider the equation $\Delta u=e^{u+e^{u}}$. In both cases, we underline the effect of the geometry of the domain in the asymptotic expansion of the solutions near the boundary $\partial \Omega$.

Copyright (c) 2006 Claudia Anedda et al. This is an open access article distributed under the Creative Commons Attribution License, which permits unrestricted use, distribution, and reproduction in any medium, provided the original work is properly cited.

\section{Introduction}

Let $\Omega \subset R^{N}$ be a bounded smooth domain. In 1916, Bieberbach [10] has investigated the problem

$$
\Delta u=e^{u} \quad \text { in } \Omega, \quad u(x) \longrightarrow \infty \quad \text { as } x \longrightarrow \partial \Omega,
$$

and has proved the existence of a classical solution called a boundary blowup (explosive, large) solution. Moreover, if $\delta=\delta(x)$ denotes the distance from $x$ to $\partial \Omega$, we have [10] $u(x)-\log \left(2 / \delta^{2}(x)\right) \rightarrow 0$ as $x \rightarrow \partial \Omega$. Recently, Bandle [4] has improved the previous estimate finding the expansion

$$
u(x)=\log \frac{2}{\delta^{2}(x)}+(N-1) K(\bar{x}) \delta(x)+o(\delta(x)),
$$

where $K(\bar{x})$ denotes the mean curvature of $\partial \Omega$ at the point $\bar{x}$ nearest to $x$, and $o(\delta)$ has the usual meaning. Boundary estimates for various nonlinearities have been discussed in several papers, see for example [1, 3, 5, 8, 13-16].

In Section 2 of the present paper we investigate boundary blowup solutions of the equation $\Delta u=e^{u|u|^{\beta-1}}$, with $\beta>0, \beta \neq 1$. We prove the estimate

$$
u(x)=\Phi(\delta)+\beta^{-1}(N-1) K(x) \delta(\Phi(\delta))^{1-\beta}+O(1) \delta(\Phi(\delta))^{1-2 \beta}
$$


2 Second-order estimates

where $\Phi(\delta)$ is defined by the equation

$$
\int_{\Phi(s)}^{\infty}(2 F(t))^{-1 / 2}=s, \quad F(t)=\int_{-\infty}^{t} e^{\tau|\tau| \beta-1} d \tau,
$$

$K(x)$ is the mean curvature of the surface $\{x \in \Omega: \delta(x)=$ constant $\}$, and $O(1)$ denotes a bounded quantity.

In Section 3 we consider boundary blowup solutions of the equation $\Delta u=e^{u+e^{u}}$. We find the estimate

$$
u(x)=\Psi(\delta)+(N-1) K(x) e^{-\Psi(\delta)} \delta+O(1) e^{-2 \Psi(\delta)} \delta,
$$

where $\Psi$ is defined by the equation

$$
\int_{\Psi(s)}^{\infty}\left(2 e^{e^{t}}-2\right)^{-1 / 2} d t=s
$$

In this paper, the distance function $\delta=\delta(x)$ plays an important role. Recall that if $\Omega$ is smooth then also $\delta(x)$ is smooth for $x$ near to $\partial \Omega$, and [12]

$$
\sum_{i=1}^{N} \delta_{x_{i}} \delta_{x_{i}}=1, \quad-\sum_{i=1}^{N} \delta_{x_{i} x_{i}}=(N-1) K=H,
$$

where $K=K(x)$ is the mean curvature of the surface $\{x \in \Omega: \delta(x)=$ constant $\}$.

The effect of the geometry of the domain in the behaviour of boundary blowup solutions for special equations has been observed in various papers, see for example, $[2,7,9$, $11]$.

\section{The equation $\Delta u=e^{u|u|^{\beta-1}}$}

In what follows we denote with $O(1)$ a bounded quantity.

Lemma 2.1. Let $\beta>0, f(s)=e^{s \mid s^{\beta-1}}, F(s)=\int_{-\infty}^{s} f(t) d t$. Then

$$
F(s) f^{\prime}(s)(f(s))^{-2}=1+O(1) s^{-\beta} .
$$

Proof. For $s>0$ we have

$$
\begin{aligned}
F(s) f^{\prime}(s)(f(s))^{-2} & =f^{\prime}(s)(f(s))^{-2} F(0)+f^{\prime}(s)(f(s))^{-2} \int_{0}^{s} f(t) d t \\
& =\beta e^{-s^{\beta}} s^{\beta-1} F(0)+e^{-s^{\beta}} \int_{0}^{s} e^{t^{\beta}} \beta t^{\beta-1} d t+\beta e^{-s^{\beta}} \int_{0}^{s} e^{t^{\beta}}\left(s^{\beta-1}-t^{\beta-1}\right) d t \\
& =\beta e^{-s^{\beta}} s^{\beta-1} F(0)+1-e^{-s^{\beta}}+\beta e^{-s^{\beta}} \int_{0}^{s} e^{t^{\beta}}\left(s^{\beta-1}-t^{\beta-1}\right) d t .
\end{aligned}
$$

We have

$$
\begin{gathered}
\lim _{s \rightarrow \infty} s^{\beta} \beta e^{-s^{\beta}} s^{\beta-1} F(0)=0, \\
\lim _{s \rightarrow \infty} s^{\beta} e^{-s^{\beta}}=0 .
\end{gathered}
$$


Moreover, using de l'Hôpital's rule we find

$$
\begin{aligned}
\lim _{s \rightarrow \infty} \frac{\beta \int_{0}^{s} e^{t^{\beta}}\left(s^{2 \beta-1}-s^{\beta} t^{\beta-1}\right) d t}{e^{s^{\beta}}} & =\lim _{s \rightarrow \infty} \frac{\int_{0}^{s} e^{t^{\beta}}\left((2 \beta-1) s^{\beta-1}-\beta t^{\beta-1}\right) d t}{e^{s^{\beta}}} \\
& =\lim _{s \rightarrow \infty} \frac{(\beta-1) e^{s^{\beta}} s^{\beta-1}+\int_{0}^{s} e^{t^{\beta}}(2 \beta-1)(\beta-1) s^{\beta-2} d t}{\beta e^{s^{\beta}} s^{\beta-1}} \\
& =\frac{\beta-1}{\beta}+(2 \beta-1)(\beta-1) \lim _{s \rightarrow \infty} \frac{\int_{0}^{s} e^{t^{\beta}} d t}{\beta e^{s^{\beta}} s} \\
& =\frac{\beta-1}{\beta}+(2 \beta-1)(\beta-1) \lim _{s \rightarrow \infty} \frac{1}{\beta\left(1+\beta s^{\beta}\right)}=\frac{\beta-1}{\beta} .
\end{aligned}
$$

The lemma follows.

Remark 2.2. If $\beta=1$, we have $F(s) f^{\prime}(s)(f(s))^{-2}=1$. We do not care of this special case because it has been discussed in [2].

Lemma 2.3. Let $\Phi=\Phi(\delta)$ be defined by

$$
\int_{\Phi(\delta)}^{\infty}(2 F(t))^{-1 / 2} d t=\delta, \quad F(t)=\int_{-\infty}^{t} f(\tau) d \tau, f(\tau)=e^{\tau|\tau|^{\beta-1}}
$$

Then

$$
-\Phi^{\prime}(\delta)=\left[1+O(1)(\Phi(\delta))^{-\beta}\right] \delta f(\Phi(\delta))
$$

Proof. By the (trivial) relation

$$
-1+2\left(1+O(1) s^{-\beta}\right)=1+O(1) s^{-\beta},
$$

using (2.1) we have

$$
-1+2 F(s) f^{\prime}(s)(f(s))^{-2}=1+O(1) s^{-\beta} .
$$

Multiplying by $(2 F(s))^{-1 / 2}$ we find

$$
\begin{gathered}
-(2 F(s))^{-1 / 2}+(2 F(s))^{1 / 2} f^{\prime}(s)(f(s))^{-2}=(2 F(s))^{-1 / 2}+O(1)(2 F(s))^{-1 / 2} s^{-\beta}, \\
-\left((2 F(s))^{1 / 2}(f(s))^{-1}\right)^{\prime}=(2 F(s))^{-1 / 2}+O(1)(2 F(s))^{-1 / 2} s^{-\beta} .
\end{gathered}
$$

Integrating on $(s, \infty)$ we get

$$
(2 F(s))^{1 / 2}(f(s))^{-1}=\int_{s}^{\infty}(2 F(t))^{-1 / 2} d t+O(1) \int_{s}^{\infty}(2 F(t))^{-1 / 2} t^{-\beta} d t .
$$


4 Second-order estimates

Using de l'Hôpital's rule we find

$$
\begin{aligned}
\lim _{s \rightarrow \infty} \frac{s^{-\beta} \int_{s}^{\infty}(2 F(t))^{-1 / 2} d t}{\int_{s}^{\infty}(2 F(t))^{-1 / 2} t^{-\beta} d t} & =\lim _{s \rightarrow \infty} \frac{(2 F(s))^{-1 / 2} s^{-\beta}+\beta s^{-\beta-1} \int_{s}^{\infty}(2 F(t))^{-1 / 2} d t}{(2 F(s))^{-1 / 2} s^{-\beta}} \\
& =1+\lim _{s \rightarrow \infty} \frac{\beta \int_{s}^{\infty}(2 F(t))^{-1 / 2} d t}{s(2 F(s))^{-1 / 2}} \\
& =1+\lim _{s \rightarrow \infty} \frac{-\beta}{1-s(2 F(s))^{-1} f(s)}=1 .
\end{aligned}
$$

In the last step we have used the limit

$$
\lim _{s \rightarrow \infty} \frac{s f(s)}{F(s)}=\infty
$$

which can be proved easily with de l'Hôpital's rule. Using (2.11), (2.10) can be rewritten as

$$
(2 F(s))^{1 / 2}(f(s))^{-1}=\int_{s}^{\infty}(2 F(t))^{-1 / 2} d t+O(1) s^{-\beta} \int_{s}^{\infty}(2 F(t))^{-1 / 2} d t
$$

Putting $s=\Phi(\delta)$ and using the equation $-\Phi^{\prime}(\delta)=(2 F(\Phi(\delta)))^{1 / 2}$, the lemma follows.

Theorem 2.4. Let $\Omega$ be a bounded smooth domain in $R^{N}, N \geq 2$, and let $\beta>0, \beta \neq 1$. If $u(x)$ is a boundary blowup solution of $\Delta u=e^{u|u|^{\beta-1}}$ in $\Omega$, then

$$
u(x)=\Phi(\delta)+\beta^{-1} H \delta(\Phi(\delta))^{1-\beta}+O(1) \delta(\Phi(\delta))^{1-2 \beta},
$$

where $\Phi(\delta)$ is defined as in (2.5), $\delta=\delta(x)$ is the distance from $x$ to $\partial \Omega$ and $H$ is defined by (1.7).

Proof. We look for a super-solution of the form

$$
w(x)=\Phi(\delta)+\beta^{-1} H \delta(\Phi(\delta))^{1-\beta}+\alpha \delta(\Phi(\delta))^{1-2 \beta},
$$

where $\alpha$ is a positive constant to be determined. Denoting by' differentiation with respect to $\delta$, we have

$$
w_{x_{i}}=\Phi^{\prime}(\delta) \delta_{x_{i}}+\beta^{-1} H_{x_{i}} \delta(\Phi(\delta))^{1-\beta}+\beta^{-1} H\left(\delta(\Phi(\delta))^{1-\beta}\right)^{\prime} \delta_{x_{i}}+\alpha\left(\delta(\Phi(\delta))^{1-2 \beta}\right)^{\prime} \delta_{x_{i}} .
$$

Using (1.7) we find

$$
\begin{aligned}
\Delta w= & \Phi^{\prime \prime}(\delta)-\Phi^{\prime}(\delta) H+\beta^{-1} \Delta H \delta(\Phi(\delta))^{1-\beta}+2 \beta^{-1} \nabla H \cdot \nabla \delta\left(\delta(\Phi(\delta))^{1-\beta}\right)^{\prime} \\
& +\beta^{-1} H\left(\delta(\Phi(\delta))^{1-\beta}\right)^{\prime \prime}-\beta^{-1} H^{2}\left(\delta(\Phi(\delta))^{1-\beta}\right)^{\prime} \\
& +\alpha\left(\delta(\Phi(\delta))^{1-2 \beta}\right)^{\prime \prime}-\alpha\left(\delta(\Phi(\delta))^{1-2 \beta}\right)^{\prime} H .
\end{aligned}
$$


With $f(\tau)=e^{\tau|\tau|^{\beta-1}}$, by $(2.5)$ we have $\Phi^{\prime \prime}(\delta)=f(\Phi)$. Often we write $\Phi$ instead of $\Phi(\delta)$ and $\Phi^{\prime}$ instead of $\Phi^{\prime}(\delta)$. Lemma 2.3 yields

$$
-\Phi^{\prime}=\left[1+O(1) \Phi^{-\beta}\right] \delta f(\Phi) .
$$

Using (2.18) and the equation $\Phi^{\prime}=-(2 F(\Phi))^{1 / 2}$ we find

$$
\begin{aligned}
\lim _{\delta \rightarrow 0} \frac{(\Phi(\delta))^{1-\beta}}{\delta(\Phi(\delta))^{-\beta} f(\Phi)} & =\lim _{\delta \rightarrow 0} \frac{\Phi}{-\Phi^{\prime}}=\lim _{\delta \rightarrow 0} \frac{\Phi}{(2 F(\Phi))^{1 / 2}} \\
& =\lim _{s \rightarrow \infty}\left(\frac{s^{2}}{2 F(s)}\right)^{1 / 2}=\lim _{s \rightarrow \infty}\left(\frac{s}{f(s)}\right)^{1 / 2}=0 .
\end{aligned}
$$

Let us write the last result as

$$
(\Phi(\delta))^{1-\beta}=o(1) \delta(\Phi(\delta))^{-\beta} f(\Phi),
$$

where $o(1)$ denotes a quantity which tends to zero as $\delta \rightarrow 0$. Using (2.18) again we find

$$
\lim _{\delta \rightarrow 0} \frac{(\Phi(\delta))^{-\beta} \Phi^{\prime}}{\delta(\Phi(\delta))^{-\beta} f(\Phi)}=-1
$$

Therefore,

$$
\begin{aligned}
\left(\delta(\Phi(\delta))^{1-\beta}\right)^{\prime} & =(\Phi(\delta))^{1-\beta}+(1-\beta) \delta(\Phi(\delta))^{-\beta} \Phi^{\prime} \\
& =o(1) \delta(\Phi(\delta))^{-\beta} f(\Phi) .
\end{aligned}
$$

Further differentiation yields

$$
\begin{aligned}
\left(\delta(\Phi(\delta))^{1-\beta}\right)^{\prime \prime}= & 2(1-\beta)(\Phi(\delta))^{-\beta} \Phi^{\prime}-\beta(1-\beta) \delta(\Phi(\delta))^{-\beta-1}\left(\Phi^{\prime}\right)^{2} \\
& +(1-\beta) \delta(\Phi(\delta))^{-\beta} f(\Phi) .
\end{aligned}
$$

Moreover, recalling (2.12) we find

$$
\lim _{\delta \rightarrow 0} \frac{\delta(\Phi(\delta))^{-\beta-1}\left(\Phi^{\prime}\right)^{2}}{\delta(\Phi(\delta))^{-\beta} f(\Phi)}=\lim _{\delta \rightarrow 0} \frac{2 F(\Phi)}{\Phi f(\Phi)}=\lim _{s \rightarrow \infty} \frac{2 F(s)}{s f(s)}=0 .
$$

Using the last result and (2.21), from (2.23) we find

$$
\left(\delta(\Phi(\delta))^{1-\beta}\right)^{\prime \prime}=O(1) \delta(\Phi(\delta))^{-\beta} f(\Phi) .
$$

Similarly, we find

$$
\begin{aligned}
& \left(\delta(\Phi(\delta))^{1-2 \beta}\right)^{\prime}=o(1) \delta(\Phi(\delta))^{-2 \beta} f(\Phi), \\
& \left(\delta(\Phi(\delta))^{1-2 \beta}\right)^{\prime \prime}=O(1) \delta(\Phi(\delta))^{-2 \beta} f(\Phi) .
\end{aligned}
$$


Denoting by $M_{1}$ a nonnegative constant independent of $\alpha$ and using (2.18), (2.20), (2.22), (2.25), (2.26), by (2.17) we get

$$
\Delta w<f(\Phi)\left[1+H \delta+M_{1} \delta \Phi^{-\beta}+\alpha M_{1} \delta \Phi^{-2 \beta}\right]
$$

On the other side, we have

$$
\begin{aligned}
f(w) & =e^{\left(\Phi+\beta^{-1} H \delta \Phi^{1-\beta}+\alpha \delta \Phi^{1-2 \beta}\right)^{\beta}} \\
& =e^{\Phi^{\beta}\left(1+\beta^{-1} H \delta \Phi^{-\beta}+\alpha \delta \Phi^{-2 \beta}\right)^{\beta}} .
\end{aligned}
$$

Let us take $\delta_{0}>0$ and $\alpha$ such that for $\left\{x \in \Omega: \delta(x)<\delta_{0}\right\}$ we have

$$
-\frac{1}{2}<\beta^{-1} H \delta(\Phi(\delta))^{-\beta}+\alpha \delta(\Phi(\delta))^{-2 \beta}<1 .
$$

Then, denoting by $M_{2}$ a nonnegative constant independent of $\alpha$ we find

$$
\begin{aligned}
f(w) & >e^{\Phi \beta\left(1+H \delta \Phi^{-\beta}+\alpha \beta \delta \Phi^{-2 \beta}-M_{2}\left(\delta \Phi^{-\beta}\right)^{2}-M_{2}\left(\alpha \delta \Phi^{-2 \beta}\right)^{2}\right)} \\
& =f(\Phi) e^{H \delta+\alpha \beta \delta \Phi^{-\beta}-M_{2} \delta^{2} \Phi^{-\beta}-M_{2}(\alpha \delta)^{2} \Phi^{-3 \beta}} \\
& >f(\Phi)\left[1+H \delta+\alpha \beta \delta \Phi^{-\beta}-M_{2} \delta^{2} \Phi^{-\beta}-M_{2}(\alpha \delta)^{2} \Phi^{-3 \beta}\right] .
\end{aligned}
$$

By (2.27) and (2.30) we find that

$$
\Delta w<f(w)
$$

when

$$
1+H \delta+M_{1} \delta \Phi^{-\beta}+\alpha M_{1} \delta \Phi^{-2 \beta}<1+H \delta+\alpha \beta \delta \Phi^{-\beta}-M_{2} \delta^{2} \Phi^{-\beta}-M_{2}(\alpha \delta)^{2} \Phi^{-3 \beta}
$$

Rearranging we find

$$
M_{1}+M_{2} \delta<\alpha\left[\beta-M_{2} \alpha \delta \Phi^{-2 \beta}-M_{1} \Phi^{-\beta}\right]
$$

We can take $\delta_{0}$ small and $\alpha$ large so that (2.33) and (2.29) hold for $\delta(x)<\delta_{0}$.

Our function $f(t)=e^{t|t|^{\beta-1}}$ is positive and increasing for all $t$, and $F(t) t^{-2}$ is increasing for large $t$. Moreover, if $G(t)=\int_{0}^{t} \sqrt{F(s)} d s$, for $a$ and $b$ such that $1<a<2<b$, we have

$$
a \frac{F(t)}{f(t)} \leq \frac{G(t)}{G^{\prime}(t)} \leq b \frac{F(t)}{f(t)} \text { for large } t .
$$

Therefore, by [7, Theorem 4(ii)] we have, for some constant $C>0$,

$$
C \delta^{2} \Phi^{\prime}(\delta)+\Phi(\delta) \leq u(x) \leq \Phi(\delta)+C \delta \Phi(\delta)
$$

Using the right-hand side of (2.35) we find

$$
w(x)-u(x) \geq \Phi(\delta)\left[\beta^{-1} H \delta(\Phi(\delta))^{-\beta}+\alpha \delta(\Phi(\delta))^{-2 \beta}-C \delta\right] .
$$


Take $\alpha$ and $\delta_{0}$ such that (2.33) holds and put $\alpha \delta_{0}\left(\Phi\left(\delta_{0}\right)\right)^{-2 \beta}=q$. Decrease $\delta_{0}$ and increase $\alpha$ so that $\alpha \delta_{0}\left(\Phi\left(\delta_{0}\right)\right)^{-\beta}=q$ and

$$
\beta^{-1} H \delta(\Phi(\delta))^{-\beta}+q-C \delta>0
$$

for $\delta(x)=\delta_{0}$. Then, $w(x) \geq u(x)$ on $\left\{x \in \Omega: \delta(x)=\delta_{0}\right\}$. When $\alpha$ is fixed, by (2.36) we get $\liminf _{x \rightarrow \partial \Omega}[w(x)-u(x)] \geq 0$. Hence, using (2.31) we find $w(x) \geq u(x)$ on $\{x \in \Omega$ : $\left.\delta(x)<\delta_{0}\right\}$.

We look for a subsolution of the form

$$
v(x)=\Phi(\delta)+\beta^{-1} H \delta(\Phi(\delta))^{1-\beta}-\alpha \delta(\Phi(\delta))^{1-2 \beta},
$$

where $\alpha$ is a positive constant to be determined. Instead of (2.27), now we find

$$
\Delta v>f(\Phi)\left[1+H \delta-M_{1} \delta \Phi^{-\beta}-\alpha M_{1} \delta \Phi^{-2 \beta}\right] .
$$

Of course, the constant $M_{1}$ in (2.39) and the constants $M_{i}$ in what follows are not necessarily the same as in the previous case.

Now we have

$$
f(v)=e^{\Phi^{\beta}\left(1+\beta^{-1} H \delta \Phi^{-\beta}-\alpha \delta \Phi^{-2 \beta}\right)^{\beta}} .
$$

Let us take $\delta_{0}>0$ and $\alpha$ such that, for $\left\{x \in \Omega: \delta(x)<\delta_{0}\right\}$ we have

$$
-\frac{1}{2}<\beta^{-1} H \delta(\Phi(\delta))^{-\beta}-\alpha \delta(\Phi(\delta))^{-2 \beta}<1
$$

Then,

$$
\begin{aligned}
f(v) & <e^{\Phi^{\beta}\left(1+H \delta \Phi^{-\beta}-\alpha \beta \delta \Phi^{-2 \beta}+M_{2}\left(\delta \Phi^{-\beta}\right)^{2}+M_{2}\left(\alpha \delta \Phi^{-2 \beta}\right)^{2}\right)} \\
& =f(\Phi) e^{H \delta-\alpha \beta \delta \Phi^{-\beta}+M_{2} \delta^{2} \Phi^{-\beta}+M_{2}(\alpha \delta)^{2} \Phi^{-3 \beta}} .
\end{aligned}
$$

In our next step, we take $\delta$ and $\alpha$ such that

$$
\alpha \delta \Phi^{-\beta}<1, \quad H \delta-\alpha \beta \delta \Phi^{-\beta}+M_{2} \delta^{2} \Phi^{-\beta}+M_{2}(\alpha \delta)^{2} \Phi^{-3 \beta}<1 .
$$

Then we find

$$
f(v)<f(\Phi)\left[1+H \delta-\alpha \beta \delta \Phi^{-\beta}+M_{3} \delta^{2}+M_{3}(\alpha \delta)^{2} \Phi^{-2 \beta}\right] .
$$

By (2.39) and (2.44) we find that $\Delta v>f(v)$ provided

$$
1+H \delta-M_{1} \delta \Phi^{-\beta}-\alpha M_{1} \delta \Phi^{-2 \beta}>1+H \delta-\alpha \beta \delta \Phi^{-\beta}+M_{3} \delta^{2}+M_{3}(\alpha \delta)^{2} \Phi^{-2 \beta} .
$$

Rearranging we have

$$
\alpha\left[\beta-M_{1} \Phi^{-\beta}-M_{3} \alpha \delta \Phi^{-\beta}\right]>M_{1}+M_{3} \delta \Phi^{\beta} .
$$

Since $\delta \Phi^{\beta} \rightarrow 0$ as $\delta \rightarrow 0$, inequality (2.46) (in addition to (2.41) and (2.43)) holds for $\delta(x)<\delta_{0}$ with suitable $\delta_{0}$ and $\alpha$. 
Using the left-hand side of (2.35) we find

$$
\begin{aligned}
v(x)-u(x) & \leq \beta^{-1} H \delta(\Phi(\delta))^{1-\beta}-\alpha \delta(\Phi(\delta))^{1-2 \beta}-C \delta^{2} \Phi^{\prime}(\delta) \\
& =(\Phi(\delta))^{1-\beta}\left[\beta^{-1} H \delta-\alpha \delta(\Phi(\delta))^{-\beta}-C \delta^{2} \Phi^{\prime}(\delta)(\Phi(\delta))^{\beta-1}\right] .
\end{aligned}
$$

Take $\alpha$ and $\delta_{0}$ such that (2.46) holds, and put $\alpha \delta_{0}\left(\Phi\left(\delta_{0}\right)\right)^{-\beta}=q$. Decrease $\delta_{0}$ and increase $\alpha$ so that $\alpha \delta_{0}\left(\Phi\left(\delta_{0}\right)\right)^{-\beta}=q$ and

$$
\beta^{-1} H \delta-q-C \delta^{2} \Phi^{\prime}(\delta)(\Phi(\delta))^{\beta-1}<0
$$

for $\delta(x)=\delta_{0}$. Note that the previous inequality holds for $\delta$ small because

$$
\lim _{\delta \rightarrow 0} \frac{\delta^{2} \Phi^{\prime}(\delta)}{(\Phi(\delta))^{1-\beta}}=0,
$$

as one can prove using Lemma 2.3 and de l'Hôpital's rule. It follows from (2.47) that $v(x) \leq u(x)$ on $\left\{x \in \Omega: \delta(x)=\delta_{0}\right\}$. By (2.47) we also find that $v(x)-u(x) \leq 0$ on $\partial \Omega$. Hence $v(x) \leq u(x)$ on $\left\{x \in \Omega: \delta(x)<\delta_{0}\right\}$. The theorem follows.

\section{The equation $\Delta u=e^{u+e^{u}}$}

Lemma 3.1. Let $f(t)=e^{t+e^{t}}, F(s)=\int_{-\infty}^{s} f(t) d t$. Then

$$
F(s) f^{\prime}(s)(f(s))^{-2}=1+O(1) e^{-s},
$$

where $O(1)$ is a bounded quantity.

Proof. By computation we find

$$
F(s) f^{\prime}(s)(f(s))^{-2}=1+e^{-s}-e^{-e^{s}}-e^{-s-e^{s}} .
$$

The lemma follows.

LEMMA 3.2. Let $f(t)$ and $F(s)$ be as in Lemma 3.1. If

$$
\int_{\Psi(\delta)}^{\infty}(2 F(s))^{-1 / 2} d s=\delta
$$

we have

$$
-\Psi^{\prime}(\delta)=\left[1+O(1) e^{-\Psi(\delta)}\right] \delta f(\Psi(\delta)) .
$$

Proof. By the (trivial) relation

$$
-1+2\left(1+O(1) e^{-s}\right)=1+O(1) e^{-s},
$$

using (3.1) we have

$$
-1+2 F(s) f^{\prime}(s)(f(s))^{-2}=1+O(1) e^{-s} .
$$


Multiplying by $(2 F(s))^{-1 / 2}$ we find

$$
\begin{gathered}
-(2 F(s))^{-1 / 2}+(2 F(s))^{1 / 2} f^{\prime}(s)(f(s))^{-2}=(2 F(s))^{-1 / 2}+O(1)(2 F(s))^{-1 / 2} e^{-s}, \\
-\left((2 F(s))^{1 / 2}(f(s))^{-1}\right)^{\prime}=(2 F(s))^{-1 / 2}+O(1)(2 F(s))^{-1 / 2} e^{-s} .
\end{gathered}
$$

Integrating on $(s, \infty)$ we get

$$
(2 F(s))^{1 / 2}(f(s))^{-1}=\int_{s}^{\infty}(2 F(t))^{-1 / 2} d t+O(1) \int_{s}^{\infty}(2 F(t))^{-1 / 2} e^{-t} d t .
$$

Using de l'Hôpital's rule we find

$$
\lim _{s \rightarrow \infty} \frac{e^{-s} \int_{s}^{\infty}(2 F(t))^{-1 / 2} d t}{\int_{s}^{\infty}(2 F(t))^{-1 / 2} e^{-t} d t}=1+\lim _{s \rightarrow \infty} \frac{\int_{s}^{\infty}(2 F(t))^{-1 / 2} d t}{(2 F(s))^{-1 / 2}}=1 .
$$

Using (3.9), (3.8) can be rewritten as

$$
(2 F(s))^{1 / 2}(f(s))^{-1}=\int_{s}^{\infty}(2 F(t))^{-1 / 2} d t+O(1) e^{-s} \int_{s}^{\infty}(2 F(t))^{-1 / 2} d t .
$$

Putting $s=\Psi(\delta)$ and recalling that $-\Psi^{\prime}(\delta)=(2 F(\Psi(\delta)))^{1 / 2}$, the lemma follows.

Theorem 3.3. Let $\Omega$ be a bounded smooth domain in $R^{N}, N \geq 2$, and let $f(t)=e^{t+e^{t}}$. If $u(x)$ is a boundary blowup solution of $\Delta u=f(u)$ in $\Omega$, then we have

$$
u(x)=\Psi+H e^{-\Psi} \delta+O(1) e^{-2 \Psi} \delta,
$$

where $\Psi=\Psi(\delta)$ is defined as in Lemma 3.2 and $H=H(x)$ is defined by (1.7).

Proof. We look for a super-solution of the form

$$
w(x)=\Psi+H e^{-\Psi} \delta+\alpha e^{-2 \Psi} \delta,
$$

where $\alpha$ is a positive constant to be determined. Denoting by' differentiation with respect to $\delta$, we have

$$
w_{x_{i}}=\Psi^{\prime} \delta_{x_{i}}+H_{x_{i}} e^{-\Psi} \delta+H\left(e^{-\Psi} \delta\right)^{\prime} \delta_{x_{i}}+\alpha\left(e^{-2 \Psi} \delta\right)^{\prime} \delta_{x_{i}} .
$$

Using (1.7) we find

$$
\begin{aligned}
\Delta w= & \Psi^{\prime \prime}-\Psi^{\prime} H+\Delta H e^{-\Psi} \delta+\left(2 \nabla H \cdot \nabla \delta-H^{2}\right)\left(e^{-\Psi} \delta\right)^{\prime}+H\left(e^{-\Psi} \delta\right)^{\prime \prime} \\
& -\alpha H\left(e^{-2 \Psi} \delta\right)^{\prime}+\alpha\left(e^{-2 \Psi} \delta\right)^{\prime \prime} .
\end{aligned}
$$

By Lemma 3.2 we have $-\Psi^{\prime}=\left[1+O(1) e^{-\Psi}\right] \delta f(\Psi)$, and $\Psi^{\prime \prime}=f(\Psi)$. Moreover, since $\Psi^{\prime} \delta \rightarrow 0$ as $\delta \rightarrow 0$, for $\delta$ small we also find

$$
0<\left(e^{-\Psi} \delta\right)^{\prime}=e^{-\Psi}-e^{-\Psi} \Psi^{\prime} \delta<C_{1} e^{-\Psi}
$$


We denote with $C_{i}$ positive constants (independent of $\alpha$ ). Since $f(\Psi) \delta^{2} \rightarrow 0$ and $f(\Psi) \delta \rightarrow$ $\infty$ as $\delta \rightarrow 0$, we get

$$
0<\left(e^{-\Psi} \delta\right)^{\prime \prime}=-2 e^{-\Psi} \Psi^{\prime}-e^{-\Psi} f(\Psi) \delta+e^{-\Psi}\left(\Psi^{\prime}\right)^{2} \delta<C_{2} e^{-\Psi} f(\Psi) \delta .
$$

Similarly, we find

$$
\begin{gathered}
0<\left(e^{-2 \Psi} \delta\right)^{\prime}<C_{3} e^{-2 \Psi}, \\
0<\left(e^{-2 \Psi} \delta\right)^{\prime \prime}<C_{4} e^{-2 \Psi} f(\Psi) \delta .
\end{gathered}
$$

Therefore, by (3.14) we infer

$$
\Delta w<f(\Psi)\left[1+H \delta+M_{1} e^{-\Psi} \delta+\alpha M_{2} e^{-2 \Psi} \delta\right] .
$$

On the other side, since

$$
e^{w}=e^{\Psi+H e^{-\Psi} \delta+\alpha e^{-2 \Psi} \delta}>e^{\Psi}\left[1+H e^{-\Psi} \delta+\alpha e^{-2 \Psi} \delta\right],
$$

we find

$$
\begin{aligned}
f(w) & =e^{w+e^{w}}>e^{\Psi+H e^{-\Psi} \delta+\alpha e^{-2 \Psi} \delta+e^{\Psi}\left[1+H e^{-\Psi} \delta+\alpha e^{-2 \Psi} \delta\right]} \\
& =e^{\Psi+e^{\Psi}} e^{\left[H e^{-\Psi} \delta+\alpha e^{-2 \Psi} \delta+H \delta+\alpha e^{-\Psi} \delta\right]} \\
& >f(\Psi)\left[1-M_{3} e^{-\Psi} \delta+H \delta+\alpha e^{-\Psi} \delta\right] .
\end{aligned}
$$

By (3.18) and (3.20) we have

$$
\Delta w<f(w)
$$

provided

$$
1+H \delta+M_{1} e^{-\Psi} \delta+\alpha M_{2} e^{-2 \Psi} \delta<1-M_{3} e^{-\Psi} \delta+H \delta+\alpha e^{-\Psi} \delta .
$$

Rearranging we find

$$
M_{1}+M_{3}<\alpha\left[1-M_{2} e^{-\Psi(\delta)}\right]
$$

Inequality (3.23) holds provided $\delta$ is small and $\alpha$ is large enough.

The function $f(t)=e^{t+e^{t}}$ is positive and increasing for all $t$. If $F(t)$ is defined as in Lemma 3.1, the function $F(t) t^{-2}$ is increasing for large $t$. Moreover, if $G(t)=\int_{0}^{t} \sqrt{F(s)} d s$, for $1<a<2<b$ we have

$$
a \frac{F(t)}{f(t)} \leq \frac{G(t)}{G^{\prime}(t)} \leq b \frac{F(t)}{f(t)} \quad \text { for large } t .
$$

Therefore, by [7, Theorem 4(ii)] we have, for some constant $C>0$,

$$
C \delta^{2} \Psi^{\prime}(\delta)+\Psi(\delta) \leq u(x) \leq \Psi(\delta)+C \delta \Psi(\delta)
$$


Using the right-hand side of (3.25) we find

$$
w(x)-u(x) \geq H e^{-\Psi} \delta+\alpha e^{-2 \Psi} \delta-C \delta \Psi(\delta) .
$$

Take $\alpha$ and $\delta_{0}$ so that (3.23) holds for $\delta(x)=\delta_{0}$ and put $q=\alpha e^{-2 \Psi\left(\delta_{0}\right)} \delta_{0}$. Decrease $\delta_{0}$ and increase $\alpha$ so that $\alpha e^{-2 \Psi\left(\delta_{0}\right)} \delta_{0}=q$ and $H e^{-\Psi} \delta+q-C \delta \Psi(\delta)>0$ for $\delta(x)=\delta_{0}$. Recall that $\delta \Psi(\delta) \rightarrow 0$ as $\delta \rightarrow 0$. Then, $w(x) \geq u(x)$ on $\left\{x \in \Omega: \delta(x)=\delta_{0}\right\}$. Moreover, by (3.26) we have $w(x)-u(x) \geq 0$ on $\partial \Omega$. Hence, using (3.21) we find $w(x) \geq u(x)$ on $\{x \in \Omega: \delta(x)<$ $\left.\delta_{0}\right\}$.

Let us prove that

$$
v=\Psi+H e^{-\Psi} \delta-\alpha e^{-2 \Psi} \delta
$$

is a subsolution provided $\alpha$ is a suitable positive constant. By computation, instead of (3.18), now we find

$$
\Delta v>f(\Psi)\left[1+H \delta-M_{4} e^{-\Psi} \delta-\alpha M_{5} e^{-2 \Psi} \delta\right]
$$

The next step is slightly delicate. Take $\alpha$ and $\delta$ such that

$$
e \alpha e^{-\Psi} \delta<1, \quad H e^{-\Psi} \delta-\alpha e^{-2 \Psi} \delta<1 .
$$

Then, using the second inequality in (3.29), we find

$$
e^{v}=e^{\Psi+H e^{-\Psi} \delta-\alpha e^{-2 \Psi} \delta}<e^{\Psi}\left[1+H e^{-\Psi} \delta-\alpha e^{-2 \Psi} \delta+e\left(H e^{-\Psi} \delta\right)^{2}+e\left(\alpha e^{-2 \Psi} \delta\right)^{2}\right] .
$$

Hence, using the first inequality in (3.29), we get

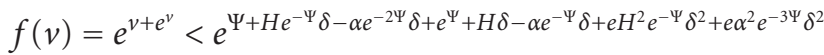

$$
\begin{aligned}
& <f(\Psi) e^{H \delta+M_{6} e^{-\Psi} \delta-\alpha e^{-\Psi} \delta}<f(\Psi)\left[1+H \delta+M_{7} e^{-\Psi} \delta-\alpha e^{-\Psi} \delta+\left(\alpha e^{-\Psi} \delta\right)^{2}\right] .
\end{aligned}
$$

Comparing the last estimate with (3.28) we have

$$
\Delta v>f(v)
$$

provided

$$
1+H \delta-M_{4} e^{-\Psi} \delta-\alpha M_{5} e^{-2 \Psi} \delta>1+H \delta+M_{7} e^{-\Psi} \delta-\alpha e^{-\Psi} \delta+\left(\alpha e^{-\Psi} \delta\right)^{2} .
$$

Rearranging, this inequality reads as

$$
\alpha\left[1-\alpha e^{-\Psi} \delta-M_{5} e^{-\Psi}\right]>M_{4}+M_{7} .
$$

Of course, (3.34) and (3.29) hold provided $\alpha$ is large and $\delta$ is small enough. Using the left-hand side of (3.25), decreasing $\delta_{0}$ and increasing $\alpha$ if necessary, one proves that $v(x)-$ $u(x) \leq 0$ at all points in $\Omega$ with $\delta(x)=\delta_{0}$. Moreover, using (3.25) again we observe that $v(x)-u(x) \leq 0$ on $\partial \Omega$. Therefore, by (3.32) it follows that $v(x)$ is a subsolution on $\{x \in$ $\left.\Omega: \delta(x)<\delta_{0}\right\}$. The theorem is proved. 


\section{References}

[1] L. Andersson and P. T. Chruściel, Solutions of the constraint equations in general relativity satisfying "hyperboloidal boundary conditions", Dissertationes Mathematicae (Rozprawy Matematyczne) 355 (1996), 1-100.

[2] C. Anedda, A. Buttu, and G. Porru, Boundary estimates for blow-up solutions of elliptic equations with exponential growth, to appear in Proceedings Differential and Difference Equations.

[3] C. Anedda and G. Porru, Higher order boundary estimates for blow-up solutions of elliptic equations, to appear in Differential Integral Equations.

[4] C. Bandle, Asymptotic behaviour of large solutions of quasilinear elliptic problems, Zeitschrift für Angewandte Mathematik und Physik 54 (2003), no. 5, 731-738.

[5] C. Bandle and E. Giarrusso, Boundary blow up for semilinear elliptic equations with nonlinear gradient terms, Advances in Differential Equations 1 (1996), no. 1, 133-150.

[6] C. Bandle and M. Marcus, "Large" solutions of semilinear elliptic equations: existence, uniqueness and asymptotic behaviour, Journal d'Analyse Mathématique 58 (1992), 9-24.

[7] __ On second-order effects in the boundary behaviour of large solutions of semilinear elliptic problems, Differential and Integral Equations 11 (1998), no. 1, 23-34.

[8] __ Dependence of blowup rate of large solutions of semilinear elliptic equations, on the curvature of the boundary, Complex Variables. Theory and Application 49 (2004), no. 7-9, 555-570.

[9] S. Berhanu and G. Porru, Qualitative and quantitative estimates for large solutions to semilinear equations, Communications in Applied Analysis 4 (2000), no. 1, 121-131.

[10] L. Bieberbach, $\Delta u=e^{u}$ und die automorphen Funktionen, Mathematische Annalen 77 (1916), no. 2, 173-212.

[11] M. del Pino and R. Letelier, The influence of domain geometry in boundary blow-up elliptic problems, Nonlinear Analysis. Theory, Methods \& Applications. Series A: Theory and Methods 48 (2002), no. 6, 897-904.

[12] D. Gilbarg and N. S. Trudinger, Elliptic Partial Differential Equations of Second Order, Grundlehren der mathematischen Wissenschaften, vol. 224, Springer, Berlin, 1977.

[13] A. Greco and G. Porru, Asymptotic estimates and convexity of large solutions to semilinear elliptic equations, Differential and Integral Equations 10 (1997), no. 2, 219-229.

[14] J. B. Keller, On solutions of $\Delta u=f(u)$, Communications on Pure and Applied Mathematics 10 (1957), 503-510.

[15] A. C. Lazer and P. J. McKenna, Asymptotic behavior of solutions of boundary blowup problems, Differential and Integral Equations 7 (1994), no. 3-4, 1001-1019.

[16] R. Osserman, On the inequality $\Delta u \geq f(u)$, Pacific Journal of Mathematics 7 (1957), no. 4, 16411647.

Claudia Anedda: Dipartimento di Matematica, Universitá di Cagliari, Via Ospedale 72,

09124 Cagliari, Italy

E-mail address: canedda@unica.it

Anna Buttu: Dipartimento di Matematica, Universitá di Cagliari, Via Ospedale 72,

09124 Cagliari, Italy

E-mail address: buttu@uncia.it

Giovanni Porru: Dipartimento di Matematica, Universitá di Cagliari, Via Ospedale 72, 09124 Cagliari, Italy

E-mail address: porru@unica.it 